\title{
REVISÃo de LITERATURA Eficácia da reabilitação cognitiva na melhoria e manutenção das atividades de vida diária em pacientes com doença de Alzheimer: uma revisão sistemática da literatura
}

\author{
Efficacy of cognitive rehabilitation in improving and maintaining daily living \\ activities in patients with Alzheimer's disease: a systematic review of literature \\ Camila de Carvalho Sá \\ https://orcid.org/0000-0002-5912-680X \\ Daiane Fuga da Silva² \\ https://orcid.org/0000-0002-9255-3694 \\ Aline Bigongiari ${ }^{1}$ \\ https://orcid.org/0000-0002-7102-3897 \\ Adriana Machado-Lima² \\ https://orcid.org/0000-0002-5741-3418
}

\section{Palavras-chave \\ Doença de Alzheimer, reabilitação cognitiva, revisão sistemática.}

\section{RESUMO}

Objetivo: Avaliar a eficácia da reabilitação cognitiva em pacientes com a doença de Alzheimer em estágio leve a moderado por meio de uma revisão sistemática. Métodos: $\bigcirc$ levantamento de artigos se deu pelas bases de dados MEDLINE (acessado via PubMed), EMBASE e LILACS, seguindo a Colaboração Cochrane e a recomendação PRISMA, por meio dos critérios de elegibilidade: ensaios clínicos randomizados e que apresentaram o uso da reabilitação cognitiva como intervenção não farmacológica em pacientes com a doença de Alzheimer em estágio leve a moderado, devendo haver pelo menos um grupo de intervenção e um grupo de comparação. Resultados: Foram selecionados seis ensaios clínicos randomizados para a realização desta revisão. Em todos os estudos analisados, foi observada melhora no desempenho dos pacientes nos instrumentos de avaliação, principalmente no que diz respeito à realização de atividades da vida diária. Conclusão: A reabilitação cognitiva é capaz de prolongar a independência do paciente na realização de atividades da vida diária e de gerar alterações funcionais e estruturais. Como consequência da constatação de poucos trabalhos que atingiram os critérios de elegibilidade, também se constatou a necessidade de se realizarem mais estudos controlados e com maior controle de aplicação, considerando o alto risco de viés nos estudos utilizados.

\section{ABSTRACT}

Objective: To evaluate the efficacy of Cognitive Rehabilitation in patients with mild to moderate Alzheimer's disease through a systematic review. Methods: The survey of articles was done by MEDLINE (accessed via PubMed), EMBASE and LILACS, following the

1 Universidade São Judas Tadeu (USJT), São Paulo, SP, Brasil.

2 Universidade São Judas Tadeu (USJT), Pós-Graduação Stricto Sensu em Ciências do Envelhecimento, São Paulo, SP, Brasil. 


\section{Keywords}

Alzheimer's disease, cognitive rehabilitation, systematic review.
Cochrane Collaboration and the PRISMA recommendation, using the following eligibility criteria: randomized clinical trials that presented the use of cognitive rehabilitation as non-pharmacological intervention in patients with mild to moderate Alzheimer's disease, with at least one intervention group and one comparison group. Results: Six randomized controlled trials were selected for this review. In all of the studies analyzed, improvement in the performance of patients in the assessment instruments was observed, mainly in relation to the performance of activities of daily living. Conclusion: Cognitive rehabilitation is able to prolong the independence of the patient in performing activities of daily living and generate functional and structural changes. As a consequence of the finding of few studies that met the eligibility criteria, we also verified the need for more controlled studies and greater application control, considering the high risk of bias in the studies used.

\section{INTRODUÇÃO}

De acordo com dados fornecidos pela Word Health Organization (WHO)', cerca de 50 milhões de pessoas vivem com demência em todo o mundo. A estimativa é que o número de pessoas com demência chegue a 82 milhões em 2030 e a 152 milhões em 2050. Entre os tipos de demência existentes, a doença de Alzheimer é atualmente a mais comum, representando cerca de 60\% a 70\% dos casos de demência no mundo ${ }^{2}$. A doença é caracterizada, principalmente, pela deterioração gradativa de funções cognitivas, com fases que evoluem em conformidade com a área cerebral afetada, estendendo-se também às funções motoras e com evolução variável ${ }^{3,4}$. Em âmbito fisiopatológico, a doença de Alzheimer está relacionada com a presença de placas extracelulares, as quais se consistem em um depósito da proteína $\beta$-amiloide e de emaranhados neurofibrilares que resultam da hiperfosforilação anormal da proteína tau ${ }^{5,6}$. Tais emaranhados conduzem, gradativamente, à morte neuronal ${ }^{7}$.

Mesmo sendo incurável, existem tratamentos farmacológicos e não farmacológicos que visam aliviar os sintomas e retardar o avanço da doença de Alzheimer. O tratamento não farmacológico deve ser pensado em âmbito multidisciplinar, visando à compensação das áreas cerebrais afetadas, bem como à estimulação biopsicossocial do paciente e de seus familiares. A escolha da terapia deve estar de acordo, principalmente, com a atual condição do paciente e com suas preferências individuais, ${ }^{8,9}$.

Entre os tratamentos não farmacológicos, há a reabilitação neuropsicológica, que visa à melhora do aproveitamento de habilidades cognitivas ainda preservadas, bem como reabilitar as que estão em declínio, envolvendo o paciente e todos que convivem com ele ${ }^{10}$. Já a reabilitação cognitiva, subcategoria da reabilitação neuropsicológica, é caracterizada como um tipo de intervenção que ocorre no contexto real do paciente, visando estimular funções cognitivas por meio de exercícios que reproduzam situações do cotidiano, com a utilização de estratégias compensatórias para proporcionar um melhor aproveitamento de funções ainda preservadas $^{11-13}$. Cabe salientar que a reabilitação cognitiva tem se voltado para o contexto individual, levando em consideração as preferências do paciente e buscando objetivos realistas e significativos $^{13}$.

A presente revisão sistemática tem como objetivo verificar, por meio da análise de ensaios clínicos randomizados, a eficácia da reabilitação cognitiva em pacientes com estágio leve a moderado da doença de Alzheimer. Especificamente, tem-se o objetivo de identificar as técnicas utilizadas na reabilitação cognitiva, bem como os seus benefícios na qualidade de vida dos pacientes com doença de Alzheimer e verificar se há alterações funcionais e/ou estruturais em decorrência da reabilitação cognitiva.

\section{MÉTODOS}

\section{Protocolo}

Esta revisão sistemática é uma avaliação de ensaios clínicos randomizados, os quais são caracterizados, principalmente, pela distribuição aleatória de participantes em grupos de intervenção e de controle. Esse tipo de estudo é considerado o padrão de excelência ou padrão-ouro entre os métodos de investigação clínica, pois é capaz de produzir evidências científicas diretas e com menor probabilidade de erro para esclarecer uma relação de causa-efeito entre dois eventos ${ }^{14}$. Para a seleção e a avaliação dos ensaios clínicos randomizados, seguiram-se dois parâmetros: a recomendação PRISMA (Principais Itens para Relatar Revisões Sistemáticas e Metanálises - do inglês Preferred Reporting Items for Systematic Reviews and Meta-Analyses) e a colaboração Cochrane.

A recomendação PRISMA consiste em um checklist com 27 itens e um fluxograma de quatro etapas ${ }^{15}$. O checklist é composto por tópicos que auxiliam no momento de elegibilidade dos estudos levantados e na construção do trabalho, sendo dividido nas seções: título, resumo, introdução racional, objetivos, protocolo e registro, critérios de elegibilidade, fontes de informação, busca, seleção dos estudos, processo de coleta de dados, lista dos dados, risco de viés em cada estudo, medidas de sumarização, síntese dos resultados, risco de viés entre estudos, análises adicionais, seleção de estudos, caracterização dos estudos, risco de viés em cada estudo, 
resultados de estudos individuais, síntese dos resultados, risco de viés entre estudos, análises adicionais, sumário da evidência, limitações, conclusões e financiamento. No que diz respeito ao fluxograma, este é dividido em: identificação, seleção, elegibilidade e inclusão, além de tratar detalhadamente todas as fases do processo da revisão sistemática, até a eleição final dos trabalhos selecionados.

A utilização da colaboração Cochrane foi por meio do Manual Cochrane para o Desenvolvimento de Revisões Sistemáticas de Intervenção, versão 5.1.0 (Cochrane Handbook) ${ }^{16}$, para descrever e detalhar a avaliação do risco de viés dos ensaios clínicos randomizados. Essa ferramenta está disponível no programa Review Manager (RevMan) ${ }^{17}$ e sua crítica avaliação é baseada em duas partes, em que estão contidos sete domínios, denominados: geração da sequência aleatória, ocultação da alocação, cegamento de participantes e profissionais, cegamento de avaliadores de desfecho, desfechos incompletos, relato de desfecho seletivo e outras fontes de vieses.

A primeira parte refere-se à descrição do que foi relatado no estudo que está sendo avaliado, em detalhes suficientes para que o julgamento seja feito com base nessas informações. A segunda parte é o julgamento quanto ao risco de viés para cada um dos domínios analisados, que podem ser classificados em três categorias: baixo risco de viés, alto risco de viés ou risco de viés incerto.

\section{Critério de elegibilidade}

Para serem incluídos na revisão, os estudos tiveram que ser ensaios clínicos randomizados e que apresentavam o uso da reabilitação cognitiva como intervenção não farmacológica em pacientes com doença de Alzheimer leve a moderada, devendo haver pelo menos um grupo de intervenção e um grupo de comparação. Dessa forma, os critérios de inclusão que foram adotados na seleção dos estudos foram: pacientes com doença de Alzheimer em estágio leve a moderado; uso das técnicas da reabilitação cognitiva como intervenção; estudos que avaliassem memória, cognição, qualidade de vida, realização de atividades da vida diária, comportamento e/ou humor, e também foram considerados aqueles que avaliassem aspectos estruturais, desde que houvesse algum tipo de relação com algum desses domínios citados. Foram excluídos os estudos que adotassem como intervenção atividades multidisciplinares ou qualquer tipo de junção entre a reabilitação cognitiva e outra intervenção não farmacológica.

\section{Estratégia de pesquisa}

As pesquisas bibliográficas foram realizadas nos seguintes bancos de dados eletrônicos: MEDLINE (acessado via PubMed), EMBASE e LILACS. Os termos de pesquisa utilizados individualmente ou combinados incluíram a reabilitação cognitiva em pacientes com a doença de Alzheimer e uma série de palavras previamente propostas, que produziu alta sensibilidade na busca por controle randomizado de ensaios clínicos. Não houve restrições de idioma na estratégia. A estratégia de pesquisa usada para o banco de dados PubMed apresenta-se no quadro 1. Os termos foram ajustados para atender aos requisitos de cada banco de dados eletrônico. Os artigos não disponíveis na íntegra foram excluídos.

\section{Seleção de estudo e extração de dados}

Duas revisoras (Camila de Carvalho Sá e Aline Bigongiari), de forma separada e independente, selecionaram os títulos e resumos dos estudos identificados na pesquisa inicial. Um checklist padronizado baseado nos critérios de elegibilidade foi utilizado para cada estudo. Estudos que não atendiam aos critérios de acordo com os títulos ou resumos foram excluídos. As versões completas dos artigos que permaneceram no estudo, incluindo os potencialmente elegíveis e incertos, foram revistos de forma independente numa segunda revisão para determinar sua elegibilidade. Desacordos em relação à elegibilidade do estudo foram discutidos entre as duas

Quadro 1. Estratégia de pesquisa utilizada para a base de dados PubMed e a estrutura PICO

População "alzheimer disease"[Mesh] OR "Disease, Alzheimer" OR "Alzheimer Sclerosis" OR

"Sclerosis, Alzheimer" OR “Alzheimer Syndrome" OR "Syndrome, Alzheimer" OR "Alzheimer Dementia (AD)" OR "Dementia, Alzheimer (AD)" OR "Alzheimer-Type Dementia (ATD)" OR "Alzheimer Type Dementia (ATD)" OR "Dementia, Alzheimer-Type (ATD)" OR "Primary Senile Degenerative Dementia" OR "Dementia, Senile" OR "Senile Dementia" OR "Dementia, Alzheimer Type" OR "Alzheimer Type Dementia" OR "Senile Dementia, Alzheimer Type" OR "Alzheimer Type Senile Dementia" OR "Dementia, Primary Senile Degenerative" OR "Alzheimer's Disease" OR "Disease, Alzheimer's" OR "Acute Confusional Senile Dementia" OR "Senile Dementia, Acute Confusional" OR "Dementia, Presenile" OR "Presenile Dementia" OR “Alzheimer Disease, Late Onset" OR "Late Onset Alzheimer Disease" OR "Alzheimer's Disease, Focal Onset" OR "Focal Onset Alzheimer's Disease" OR "Familial

Alzheimer Disease (FAD)" OR "Alzheimer Disease, Early Onset" OR "Early Onset Alzheimer Disease" OR "Presenile Alzheimer Dementia"

Intervenção "rehabilitation"[Mesh] OR "Habilitation" AND "Cognition" [Mesh] $O R$ "cognitions" $O R$ "Cognitive Function" $O R$ "Cognitive Functions" OR "Function, Cognitive" OR "Functions, Cognitive"

Desfecho "cognition"[MeSH] OR "Cognitions" OR "Cognitive Function" OR "Cognitive Functions" OR "Function, Cognitive" OR "Functions, Cognitive" $O R$ "activities of daily living"[MeSH] OR "ADL" OR "Activities, Daily Living" OR "Activity, Daily Living" OR "Daily Living Activities" OR "Daily Living Activity" OR "Living Activities, Daily" OR "Living Activity, Daily" OR "Limitation of Activity, Chronic" OR "Chronic Limitation of Activity" OR "Self Care (Rehabilitation)" OR "Care, Self (Rehabilitation)" OR "Cares, Self (Rehabilitation)" OR "Self Cares (Rehabilitation)"

Tipo de $\quad(($ randomized controlled trial[pt] OR controlled clinical trial[pt] estudo $\quad O R$ randomized[tiab] $O R$ placebo[tiab] $O R$ drug therapy[sh] $O R$ randomly[tiab] OR trial[tiab] OR groups[tiab] NOT (animals [mh] NOT humans $[\mathrm{mh}])))$ ) 
revisoras. Os seguintes dados foram extraídos de estudos inclusos: desenho metodológico, número de participantes, grupo(s) de comparação, protocolos de intervenção e resultados. O resultado primário extraído foi a eficácia da reabilitação cognitiva e pacientes com doença de Alzheimer. Duas autoras de revisão (Camila de Carvalho Sá e Aline Bigongiari), separadamente, extraíram os dados de forma independente, e desentendimentos em relação à extração de dados foram resolvidos por discussão.

\section{Avaliação de risco de viés}

Duas autoras da revisão (Camila de Carvalho Sá e Aline Bigongiari) avaliaram, de forma independente, o risco de viés dos estudos incluídos, considerando os itens estabelecidos na ferramenta de Colaboração da Cochrane para avaliar o risco de viés dos ensaios clínicos randomizados: geração adequada de sequências, ocultação de alocação, cegamento de pacientes e pesquisadores, cegamento dos avaliadores de resultados, descrição de perdas e exclusões e análise de intenção de tratar.

\section{RESULTADOS}

\section{Descrição dos estudos}

A partir dos 478 artigos potencialmente relevantes obtidos nos bancos de dados eletrônicos PubMed, EMBASE e LILACS, foram primeiramente removidos por duplicidade 105 artigos. Depois de realizada a leitura de títulos e resumos, foram excluídos 341 artigos. Dos 10 artigos restantes para leitura, ainda foram excluídos quatro artigos, devido aos seguintes motivos: não havia o texto completo disponível nas bases de dados (3); utilização da estimulação cognitiva como intervenção não farmacológica (1). Finalmente, restaram seis artigos para a revisão sistemática, conforme diagrama do fluxograma de artigos (Figura 1).

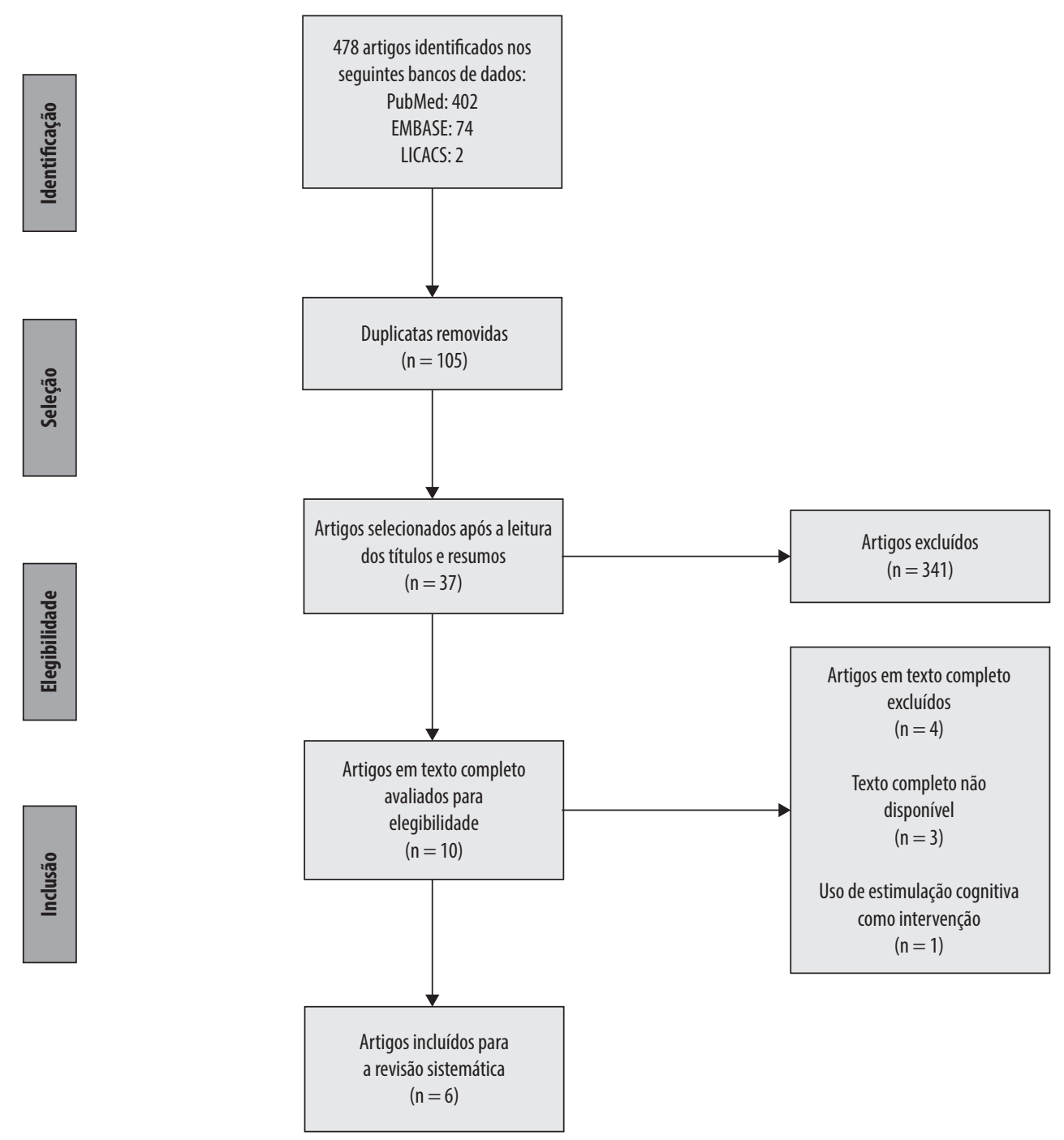

Figura 1. Diagrama do fluxograma de artigos selecionados sobre a reabilitação cognitiva em pacientes com a doença de Alzheimer. 


\section{Risco de viés}

A análise do risco de viés (Figura 2) foi realizada nos seis trabaIhos elegidos, por meio de itens como: geração adequada de sequências (27\%), ocultação de alocação (23\%), cegamento de pacientes e pesquisadores (30\%), cegamento dos avaliadores de resultado (82\%), descrição de perdas e exclusões (82\%), relato de desfecho seletivo (100\%) e análise de outros vieses (70\%).

\section{Efeitos das intervenções}

A seguir (Quadro 2), serão apresentados os seis ensaios clínicos selecionados para a realização desta revisão, incluindo os resultados observados nos grupos intervenção após o uso da reabilitação cognitiva como método interventivo em pacientes com a doença de Alzheimer em estágio leve a moderado. Dos seis estudos analisados, três ${ }^{19-21}$ realizaram a ministração de inibidores da acetilcolinesterase nos grupos controle e de intervenção.

Com relação às intervenções aplicadas nos participantes alocados no grupo intervenção, cabe salientar que, na maioria dos estudos selecionados, o programa de reabilitação cognitiva foi composto por atividades orientadas às me-

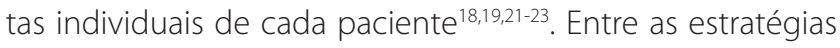
e atividades utilizadas, pode-se citar a aprendizagem sem erro ${ }^{18,20,22,23}$, recuperação espaçada' ${ }^{18,23}$, mnemônicos verbais e visuais ${ }^{19}$, treino para o aprendizado ou reaprendizado de atividades da vida diária18-20,23, gerenciamento de estresse ${ }^{21}$, bem como a utilização de estratégias compensatórias, por meio do uso de calendários e diários ${ }^{20}$.

\section{DISCUSSÃO}

Em conformidade com a afirmação de Gomes ${ }^{13}$, notou-se uma tendência à realização de programas individuais de reabilitação cognitiva, em que são priorizadas as metas individuais do indivíduo. Nesse modelo, há maior diálogo entre terapeuta e paciente no planejamento de metas e, consequentemente, nas atividades a serem realizadas.

Como exemplo, cabe destacar o estudo de Amieva et al. ${ }^{22}$, que utilizou uma amostra significativa para comparar a eficácia de um programa individual de reabilitação cognitiva (157), de treino cognitivo (170) e do uso da técnica de reminiscência (172), além de alocar 154 pessoas em um grupo controle, no qual nenhuma intervenção não farmacológica foi feita. Como resultado dessa comparação, Amieva et al. (2015) observaram que a maior melhora clínica foi encontrada nos pacientes que fizeram parte da reabilitação cognitiva, sendo também notado menor declínio funcional nesse grupo após o $24^{\circ}$ mês de acompanhamento quando comparado aos demais grupos.

Nessa mesma proposta de intervenção, o ensaio clínico de Clare et al. ${ }^{21}$ também demonstrou resultados positivos nos níveis estrutural e funcional, visto que, nos pacientes que participaram da reabilitação cognitiva em formato individual e orientado por metas, foi observada melhor performance e satisfação na realização das atividades escolhidas para o atingimento das metas. Além disso, notou-se melhora na oxigenação cerebral desses mesmos pacientes, indicando um aumento da atividade cerebral.

Levando em consideração o tamanho da amostra do estudo de Amieva et al. ${ }^{22}$, juntamente com os resultados observados em seu estudo e no de Clare et al. ${ }^{21}$, nota-se um forte indício de que a reabilitação cognitiva em formato individual e orientado para as metas do paciente é eficaz para a promoção de melhoras funcionais, além de se mostrar como uma forma de engajar o paciente em seu tratamento, visto que ele terá maior participação e consciência do processo de intervenção como um todo.

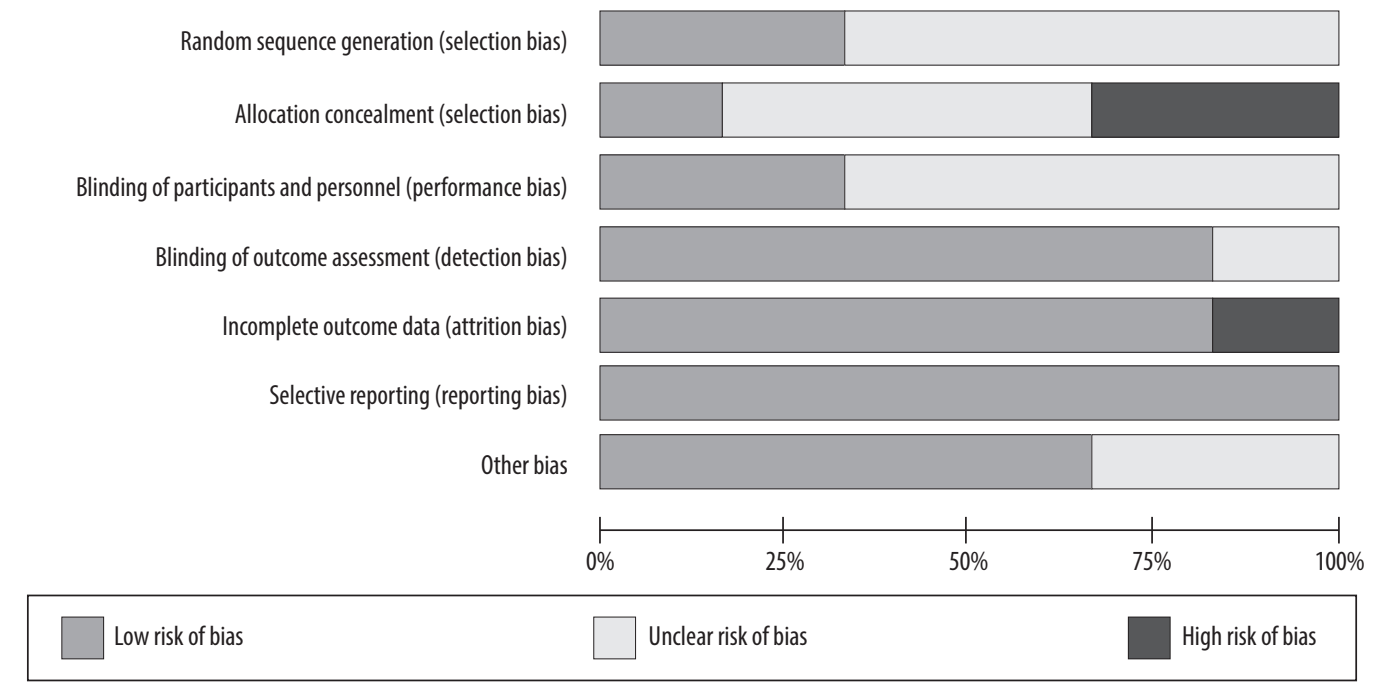

Figura 2. Diagrama de análise de risco de viés dos artigos selecionados sobre a reabilitação cognitiva em pacientes com a doença de Alzheimer. 
Quadro 2. Comparação de ensaios clínicos randomizados sobre a reabilitação cognitiva em pacientes com a doença de Alzheimer

\begin{tabular}{|c|c|c|c|}
\hline Autor & Objetivos & Amostra & Instrumentos para avaliação \\
\hline $\begin{array}{l}\text { Thivierg } \\
\text { et al. }{ }^{18}\end{array}$ & $\begin{array}{l}\text { Investigar a eficácia } \\
\text { de um programa de } \\
\text { reabilitação cognitiva } \\
\text { orientado por metas } \\
\text { no reaprendizado } \\
\text { de atividades } \\
\text { instrumentais da vida } \\
\text { diária. }\end{array}$ & $\begin{array}{l}\mathrm{N}=9 \\
\text { (reabilitação } \\
\text { cognitiva); } \\
\mathrm{N}=8 \text { (grupo } \\
\text { controle) }\end{array}$ & $\begin{array}{l}\text { Mini-Mental State Examination (MMSE); } \\
\text { Dementia Rating Scale-2 (DRS-2); } \\
\text { California Verbal Learning Test-2; } \\
\text { Clock Drawing Test e Free Drawing and } \\
\text { Copy; Lexical and Semantic Fluency } \\
\text { Test; Boston Naming Test; Trail Making } \\
\text { Test; Wechsler Adult Intelligence } \\
\text { Scale (WAIS-III); Evaluation of Praxis } \\
\text { Abilities; Hamilton Depression Rating } \\
\text { Scale; Neuropsychiatric Inventory (NPI); } \\
\text { Disability Assessment for Dementia } \\
\text { (DAD); Direct Measure of Training } \\
\text { (DMT); Rivermead Behavioural Memory } \\
\text { Test (RBMT); Dementia Quality of Life } \\
\text { questionnaire (DQoL) }\end{array}$ \\
\hline $\begin{array}{l}\text { Paassch } \\
\text { en et al. }{ }^{19}\end{array}$ & $\begin{array}{l}\text { Avaliar as possíveis } \\
\text { mudanças nos } \\
\text { padrões de ativação } \\
\text { cerebral após a } \\
\text { intervenção. }\end{array}$ & $\begin{array}{l}\mathrm{N}=7 \\
\text { (reabilitação } \\
\text { cognitiva); } \\
\mathrm{N}=12 \text { (grupo } \\
\text { controle) }\end{array}$ & $\begin{array}{l}\text { scanner Philips de 1,5-T; Canadian } \\
\text { Occupational Performance Measure } \\
\text { (COPM); Hospital Anxiety and } \\
\text { Depression Scale (HADS); } \\
\text { Functional Magnetic Resonance Imaging } \\
\text { (fMRI paradigma) }\end{array}$ \\
\hline
\end{tabular}

Bottino et al. ${ }^{20}$

Amieva et al. 22

Haman et al. ${ }^{23}$

Clare et al. ${ }^{21}$
Avaliar 0 impacto da reabilitação cognitiva em sintomas comportamentais e psicológicos de pacientes com doença de Alzheimer.

Verificar a eficácia da $\quad \mathrm{N}=6$ reabilitação cognitiva (reabilitação uso de inibidores da $\quad \mathrm{N}=7$ (grupo acetilcolinesterase em controle) funções cognitivas, sintomas psiquiátricos e atividades da vida diária.

\section{Comparar a eficácia} entre 0 treino cognitivo, a terapia da reminiscência e a reabilitação cognitiva em formato individual em pacientes com doença de Alzheimer.

\section{Verificar se a} reabilitação cognitiva promove melhora na performance em áreas-alvo de atividades da vida diária. $\mathrm{N}=172$ (técnica da reminiscên (ia); $\mathrm{N}=157$ (reabilitação cognitiva); controle)

$\mathrm{N}=7$

(reabilitação cognitiva); $\mathrm{N}=8$ (grupo controle)

\section{$\mathrm{N}=21$} (reabilitação cognitiva); $\mathrm{N}=23$ controle) combinada com $0 \quad$ cognitiva):
$\mathrm{N}=170$ (treino MMSE; ADAScog; NPI; DAD; Grille cognitivo); d'Autonomie Gérontologique-Groupes

$$
\mathrm{N}=154 \text { (grupo }
$$

$\mathrm{NPI} ; \mathrm{ZBI}-22$ Iso-Ressources (AGGIR); Apathy Inventory (Al); Montgomery-Asberg Depression Rating Scale (MADRS); Q $0 \mathrm{~L}-$ AD; Zarit Burden Interview (ZBI-22); Resource Utilization (RUD Lite) Wechsler Intelligence Revised Scale (WAIS-R); Verbal Fluency Semantic; Boston Naming Test (BNT); Fuld Object Memory Evaluation (FOME) (relaxame nto); Everyday Attention; Independent Living $\mathrm{N}=21$ (grupo
COPM; Rivermead Behavioral Memory test Il; verbal fluency; map search, elevator counting, and elevator counting individuais do paciente, com o uso with distraction subtests from the Test of de tarefas práticas e estratégias

Reabilitação cognitiva em sessões individuais para aprender/ reaprender uma atividade instrumental da vida diária. 0 programa envolveu 0 uso da técnica da aprendizagem sem erro e da recuperação espaçada.

Reabilitação cognitiva em sessões para aprender novas informaçōes, além de praticar a manutenção da atenção e concentração, bem como técnicas de gerenciamento de estresse.
Resultados

Houve melhora na performance em relação à realização de atividades instrumentais da vida diária. As melhorias foram mantidas por um período de 3 meses. No entanto, a intervenção não causou efeitos sobre quaisquer outras medidas.
Após o tratamento, o grupo de intervenção apresentou maior ativação cerebral durante o reconhecimento de pares de nomes e faces no meio esquerdo e no giro frontal inferior, na ínsula esquerda e nas duas regiões no córtex parietal mediano direito, indicando uma restauração parcial de áreas frontais do cérebro que estavam menos comprometidas na fase inicial da doença de Alzheimer, a qual impactou positivamente no processo de reconhecimento. 0 grupo controle mostrou diminuição da ativação nessas áreas durante o reconhecimento após o período de intervenção. 0 desempenho comportamental na aprendizagem de nomes faciais não melhorou para nenhum dos dois grupos.

Escores do MMSE ( $p=0,047)$ e backward digit span scores $(p=0,018)$ foram significativamente diferentes entre os grupos em acompanhamento. 0 grupo de intervenção mostrou melhor resultado sobre os testes cognitivos e neuropsicológicos após o programa, sendo notada certa estabilização cognitiva e funcional dos déficits dos pacientes.

No decorrer dos 24 meses de acompanhamento e avaliações, 0 grupo que realizou a reabilitação cognitiva apresentou significativamente menor declínio funcional quando comparado aos demais grupos, tendo menor probabilidade de ser institucionalizado em todos os períodos de avaliações.
A reabilitação cognitiva produziu melhora significativa nas classificações de desempenho e satisfação de metas, enquanto os escores nos outros dois grupos não mudaram. As mudanças comportamentais no grupo de reabilitação cognitiva foram suportadas por dados do fMRI para um subconjunto de participantes, em que foram observadas mudanças na ativação cerebral, bem como aumento na oxigenação cerebral durante a codificação e o reconhecimento enquanto realizavam a tarefa de reconhecimento de nomes e faces. 
Outro ponto a ser observado refere-se à combinação entre a reabilitação cognitiva e o tratamento farmacológico em três dos seis estudos analisados ${ }^{19-21}$, nos quais se demonstraram resultados positivos tanto no que diz respeito à ativação cerebral quanto à performance em avaliações funcionais. Dessa forma, pode-se intuir que a combinação entre o tratamento farmacológico e o não farmacológico pode promover uma maximização dos benefícios ao paciente e, consequentemente, viabilizar uma maior lentificação em relação ao avanço da doença.

Também cabe citar o uso da técnica da aprendizagem sem erro em quatro dos seis artigos analisados $18,20,22,23$, a qual se mostrou como um relevante recurso da reabilitação cognitiva, tendo participação significativa nos resultados positivos dos ensaios clínicos analisados. A aprendizagem sem erro é descrita por Bottino et al. ${ }^{20}$ como uma técnica que, por meio de um trabalho colaborativo e gradativo, visa promover respostas corretas e o esquecimento de memorizações erradas durante os procedimentos aplicados.

Os achados desta revisão coincidem com os resultados do estudo de Olazarán et al. ${ }^{24}$, que, por meio de uma revisão e categorização de todas as intervenções não farmacológicas existentes para o tratamento de pacientes com doença de Alzheimer, identificou a reabilitação cognitiva como intervenção mais eficaz para o retardo do avanço da doença, apesar da constatação da existência de poucos artigos sobre o tema.

Entretanto, é importante salientar que nem todos os estudos apresentaram diferenças significativas entre o grupo controle e intervenção. Nesse âmbito, cabe citar o resultado encontrado no estudo de Thivierge et al. ${ }^{18}$, em que não foram observadas melhoras significativas na memória e em funções cognitivas de modo geral. De acordo com os próprios autores, o resultado assemelhou-se aos achados de outros estudos relacionados à reabilitação cognitiva, em que os benefícios associados ao treino são encontrados especificamente no material usado para a intervenção. No entanto, ainda nesse estudo, foi observada melhora significativa no reaprendizado de atividades instrumentais da vida diária, indicando a associação da reabilitação cognitiva a prováveis benefícios ao paciente com doença de Alzheimer, principalmente no que se refere à prolongação de sua independência na realização de atividades da vida diária.

Em relação à qualidade metodológica dos estudos utilizados nesta revisão, notou-se a predominância de incerteza quanto ao risco de viés quando analisado os seguintes domínios: geração adequada de sequências (27\%), ocultação de alocação (23\%) e cegamento de pacientes e pesquisadores (30\%). A principal razão para tal constatação refere-se à falta de informações nos artigos que permitissem o julgamento.

Observou-se alto risco de viés no método utilizado para a ocultação de alocação do ensaio clínico de Brunelle-Hamann et al. ${ }^{23}$ e de Thivierge et al. ${ }^{18}$, pois, em ambos os estudos, foi utilizada a alternância dos participantes recrutados no processo de geração de sequência aleatória. Além disso, o estudo de Thivierge et al. ${ }^{18}$ apresentou alto risco de viés quando analisada a descrição de perdas e exclusões, devido às razões para a perda de alguns participantes no decorrer da pesquisa (alto nível de ansiedade de participante do Grupo 1; alto nível de carga do cuidador de participante do Grupo 2; insatisfação de participante do Grupo 2 a respeito do processo de randomização).

Outro ponto limitante para esta revisão refere-se à existência de poucos ensaios clínicos randomizados sobre a presente temática, assim como observado por Olazarán et al..24. Além disso, notou-se que a maior parte dos artigos encontrados utilizou uma amostra consideravelmente pequena para a aplicação da intervenção, limitando a viabilização de generalizações quanto aos benefícios da reabilitação cognitiva em pacientes com doença de Alzheimer.

\section{CONCLUSÕES}

Com os resultados obtidos nesta revisão, conclui-se que a reabilitação cognitiva é considerada uma intervenção eficaz para pacientes com doença de Alzheimer em estágio leve a moderado. Apesar de não trazer uma completa recuperação das funções cognitivas perdidas, ela se tornou um importante fator para a promoção de qualidade de vida aos pacientes, auxiliando na prolongação da independência na realização de atividades da vida diária.

Notou-se também a predominância da reabilitação cognitiva em formato individual e orientado para as metas individuais do paciente, tendo resultados significativamente positivos já constatados. Outro recurso utilizado na maioria dos ensaios clínicos analisados foi a técnica da aprendizagem sem erro, a qual demonstrou ser um componente importante na garantia do aprendizado e/ou reaprendizado de atividades, conforme propostas durante o programa de reabilitação.

Apesar do baixo número de artigos utilizados nesta revisão, houve a preocupação em zelar pela similaridade entre os objetivos e os recursos utilizados para a intervenção. Dessa forma, é possível concluir que a reabilitação cognitiva é benéfica e capaz de promover alterações funcionais e estruturais em pacientes com a doença de Alzheimer em estágio leve a moderado.

\section{CONTRIBUIÇÕES INDIVIDUAIS}

Todas as autoras contribuíram na busca de artigos nas bases de dados, bem como na análise dos resultados. Contribuíram também na escrita, revisão e aprovação da versão final do artigo. 


\section{CONFLITO DE INTERESSES}

Todas as autoras do presente estudo declaram não haver conflito de interesses em relação a esta revisão.

\section{REFERÊNCIAS}

1. Word Health Organization (WHO). Dementia. Disponivel em: http://www.who.int/en/ news-room/fact-sheets/detail/dementia. Acesso em: 17 jul. 2018.

2. Word Health Organization (WHO). Towards a dementia plan: a WHO guide. Geneva: World Health Organization; 2018.

3. Facture NO, Castro LAG, Menezes MCL. Doença de Alzheimer, relação entre o tempo de doença e seu estadiamento. Arq Neuropsiquiatr. 1993;51(2):175-8.

4. Sereniki A, Vital MABF. A doença de Alzheimer: aspectos fisiopatológicos e farmacológicos. Rev Psiquiatr Rio Gd Sul. 2008;30(1 Suppl).

5. Pereira PMCM. Doença de Alzheimer: perspectivas de tratamento [dissertação]. Covilhã: Universidade da Beira Interior; 2013.

6. Nitrini R, Takada LT. Fisiopatologia da doença de Alzheimer. In: BruckiSMD, Magaldi RM, Morillo LS, Carvalho I, Perroco TR, Bottino CMC, et al. (Eds.). Demências - enfoque multidisciplinar: das bases fisiopatológicas ao diagnóstico e tratamento. São Paulo: Atheneu; 2011.

7. Ferreira DC, Mainardes SCC. Doença de Alzheimer: como identificar, prevenir e tratar. In: VI Mostra Interna de Trabalhos de Iniciação Científica, 2012; Maringá. Anais. Maringá: Cesumar; 2012.

8. Bottino CMC, Carvalho IAM, Alvarez AMMA, Avila R, Zukauskas PR, Bustamante SEZ. Reabilitação cognitiva em pacientes com a doença de Alzheimer. Arq Neuropsiquiatr. 2002;60(1):70-9.

9. Silva CB, Souza EM. A demência de Alzheimer e suas terapêuticas não farmacológicas: um estudo sobre as estratégias e as intervenções em reabilitação neuropsicológica. Cad Discente. 2014;1(1).

10. Lima JS. Envelhecimento, demência e doença de Alzheimer: o que a psicologia tem a ver com isso? Rev Ciênc Hum (Florianópolis). 2006;1(40):469-89.

11. Nascimento DB, Carvalho GFJ, Costa, RMEM. ReabRA: Reabilitação Cognitiva através de uma aplicação de realidade aumentada. In: 50 Workshop de Realidade Virtual e Aumentada, 2008; Bauru: Universidade Estadual Paulista; 2008.
12. Ávila R. Resultados da reabilitação neuropsicológica em paciente com doença de Alzheimer leve. Rev Psiquiatr Clín. 2003;30(4):139-46.

13. Gomes JA. Reabilitação cognitiva no comprometimento cognitivo leve e nas demências. In: Miotto EC. Reabilitação neuropsicológica e intervenções comportamentais. Rio de Janeiro: Roca; 2016.

14. Pereira MG. Epidemiologia: teoria e prática. Rio de Janeiro: Guanabara Koogan; 2008.

15. Moher D, Liberati A, Tetzlaff J, Altman DG. Principais itens para relatar revisões sistemáticas e metanálises: a recomendação PRISMA. Epidemiol Serv Saúde. 2015;24(2):335-42.

16. Higgins JPT, Green S. Cochrane handbook for systematic reviews of interventions. The Cochrane Collaboration; 2011.

17. Review Manager (RevMan) [Computer Program. Copenhagen: The Nordic Cochrane Centre. Melbourne: The Cochrane Collaboration, 2011.

18. Thivierge S, Jean L, Simard M. A randomized cross-over controlled study on cognitive rehabilitation of instrumental activities of daily living in Alzheimer disease. Am J Geriatr Psychiatry. 2014;22(11):1188-99.

19. Paasschen JV, Clare L, Yuen KSL, Woods RT, Evans SJ, Parkinson CH, et al. Cognitive rehabilitation changes memory-related brain activity in people with Alzheimer disease. Neurorehabil Neural Repair. 2013;27(5):448-59.

20. Bottino CM, Carvalho IA, Alvarez AM, Avila R, Zukauskas PR, Bustamante SE, et al. Cognitive rehabilitation combined with drug treatment in Alzheimer's disease patients: a pilot study. Clin Rehabil. 2005;19(8):861-9.

21. Clare L, Linden DEJ, Phil D, Woods RT, Whitaker R, Evans SJ, et al. Goal-oriented cognitive rehabilitation for people with early-stage Alzheimer disease: a single-blind randomized controlled trial of clinical efficacy. Am J Geriatr Psychiatry. 2010;18(10):928-39.

22. Amieva H, Robert PH, Grandoulier AS, Meillon C, Rotrou JD, Andrieu S, et al. Group and individual cognitive therapies in Alzheimer's disease: the ETNA3 randomized trial. Int Psychogeriatr. 2015;28(5):707-17.

23. Brunelle-Hamann L, Thivierge S, Simard M. Impact of a cognitive rehabilitation intervention on neuropsychiatric symptoms in mild to moderate Alzheimer's disease. Neuropsychol Rehabil. 2015;25(5):677-707.

24. Olazarán J, Reisberg B, Clare L, Cruz I, Peña-Casanova J, Del Ser T, et al. Nonpharmacological therapies in Alzheimer's disease: a systematic review of efficacy. Dement Geriatr Cogn Disord. 2010;30(2):161-78. 